\title{
Staphylococcus intermedius
}

National Cancer Institute

\section{Source}

National Cancer Institute. Staphylococcus intermedius. NCI Thesaurus. Code C86764.

A species of facultatively anaerobic, Gram positive, cocci shaped bacteria in the phylum

Firmicutes. This species is positive for coagulase, alkaline phosphatase, catalase and

urease and negative for oxidase. It can ferment glucose, fructose, sucrose, mannose,

trehalose, and glycerol but not arabinose, maltose, or xylose. S. intermedius is

commensal of oral, nasal, and skin flora of dogs and other animals and is an

opportunistic pathogen in both animals and humans. 